\title{
Lack of reliable evidence of the effectiveness of remineralising agents for the treatment of post orthodontic white spot lesions
}

\author{
Abstracted from \\ Chen H, Liu X, Dai J, Jiang Z, Guo T, Ding Y. \\ Effect of remineralizing agents on white spot lesions after orthodontic treatment: \\ a systematic review. Am J Orthod Dentofacial Orthop 2013; 143: 376-382. \\ Address for correspondence: Yin Ding, Department of Orthodontics, School of Stomatology, Fourth Military Medical University, \\ No.17, Changle West Road, Xi'an, Shaanxi, China, 710032. E-mail: Dingyin@fmmu.edu.cn
}

\section{Question: Are remineralising agents effective in the treatment of post orthodontic white spot lesions?}

Data sources PubMed, Medline, Web of Science and the Cochrane Library databases were searched.

Study selection Selection was conducted independently by two reviewers, only randomised controlled trials were included. Study quality was assessed using the Cochrane risk of bias approach. Data extraction and synthesis Data abstraction was carried out independently by two reviewers and qualitative summary presented, because of the heterogeneity of the interventions and outcome measures. Results Seven studies were included, all except one (of medium risk) were considered to be at high risk of bias. Three studies evaluated fluoride preparations (50-ppm sodium fluoride mouth rinse, 5\% sodium fluoride varnish and $0.5 \%$ sodium fluoride chewing sticks). Four compared remineralising agents containing casein phosphopeptide amorphous calcium phosphate or casein phosphopeptide amorphous calcium fluoride phosphate.

Conclusions Based on the literature, there is a lack of reliable evidence to support the effectiveness of remineralising agents for the treatment of post orthodontic white spot lesions.

\section{Commentary}

The excitement of debonding and fixed appliance removal is so easily defeated by the appearance of white spot lesions which destroy the enthusiasm of both the orthodontist and the patient. For all the improvements in delivery of preventive oral health care, the sword of Damocles of WSLs still hangs over the process of fixed orthodontic therapy. There is a presumptive line of thought that the removal of appliance and the restoration of normal oral hygiene would lead to a natural remineralisation. From the perspective of providing evidence on the management of post orthodontic lesions, the present systematic review presents a unique perspective as all the previous evidence relates to the prevention of such lesions. The review has a precise focus in this regard.

The methodology of the review is sound and conforms to the Cochrane oral health group's handbook for systematic review of interventions. The authors have well-defined inclusion criteria which clearly support the primary aim of the study. A fairly comprehensive database search across major sources has been documented. The reviewers have maintained an objective focus and the elimination of bias by stipulating the process of agreement amongst the reviewers. The methodology and quality of the studies have been assessed, and the stringency of the process is mirrored in the flow diagram where only a possible seven studies have been included in the review.

It is here that the dilemma of constructive search for direct evidence gets complex and the methodological variability between studies does not permit the pooling of data and a subsequent metaanalysis. There is a dissonance in the controls and randomisation, and the interventions' three studies evaluate the effects of fluoride preparations of varying strengths and formulation. The other four studies study the effects of casein phosphopeptide amorphous calcium phosphate or casein phosphopeptide amorphous calcium fluoride phosphate. It is only apt that the reviewers have grouped the studies into three groups based on the interventions. The absence of effects of different agents used may be more than just ineffectiveness of these agents, but also the sample size. The visual impact of WSLs is a matter of concern and visual systems of assessment would be relevant. The severity of the WSL is a serious issue in terms of response to remineralisation and this may not be so easily discernible from visual assessment. A host of questions remain unanswered and the reader of this systematic review will be naturally curious to find answers.

From the perspective of a meticulous well structured systematic review, the present study leads by example. The conclusion is self 
evident that there is lack of reliable data to support the effectiveness of remineralising agents in treatment of post orthodontic WSLs. Better designed studies are warranted to answer some crucial questions. Yet the clinical pointers are hidden somewhere within the body of this impressive review.

\section{Anmol S Kalha}

I.T.S. Dental College, Hospital and Research Center, Greater Noida, NCR, India.

1. Guzmán-Armstrong S, Chalmers J, Warren JJ. Ask us. White Spot lesions: Prevention and Treatment. Am J Orthod Dentofacial Orthop 2010; 138: 690-696.

2. Maxfield BI, Hamdan AM, Tüfekçi E, Shroff B, Best AM, Lindauer SJ. Development of white spot lesions during orthodontic treatment: perceptions of patients, parents, orthodontists, and general dentists. Am J Orthod Dentofacial Orthop 2012; 141: 337-344.

\section{Practice point}

- It is crucial to establish a caries risk assessment protocol before, during and after treatment to permit natural processes of remineralisation and diminish the impact of WSLS

- The use of a remineralisation agent such as casein phosphopeptide amorphous calcium phosphate and fluoride might promote remineralisation, so if confronted with a WSL, even in the absence of absolutely pointed evidence, it may be prudent to use an agent in the interest of greater good of the patient.

Evidence-Based Dentistry (2013) 14, 76-77. doi:10.1038/sj.ebd.6400949 